solving hæmatosine, it ought in all cases to have this colour.

$I$ beg to assure the doctor that $I$ have not been guilty of so great an indecency as the perpetration of a pun on a subject so important as the definition of serum. I did, indeed, express myself in a somewhat jocular nanner, but without any wish to hurt his feelings.

I have passed over several arguments advanced in support of his opinions, bat $I$ fear I have already too large a space in the columns of your valuable Journal. Nor have $I$ noticed his experiments, for they appear to have been too carelessly made to require attention on my part.

I have now done with Dr. Williams : but before I bid him farewell, I cannot refrain from paying the tribute of my admiration to the beautiful perspicuity of his style, and the elegance of his diction as a writer; to his acuteness as a logiciau, and his profound acquirements as a physiologist. He has applied to me the epithets "learned critic" and "erudite reviewer." I disclaim all pretension to such high titles. I leave thern to be enjoyed by men whose ambition is greater than my own; hy half-incubated physicians and incipient authors. I lay claim to no more distinguished hononr than that of being, Sir, your very obedient servant,

April, 1841. An Apothecary.

\section{CASE OF ENLARGED THYMUS GLAND.}

By Dr. Mantell, LL.D., F.R.S.

\section{To the Editor of The Lancet.}

SIR :-The perusal of the paper headed "Thymic Asthma," in your last Number, reminded me of a case which occurred in my practice when surgeon of the Royal Ordnance Hospital, at Ringmer, and is detailed in the following extract from my note. book, which I beg to transfer to the pages of The Lancer. I have the honour to be, Sir, \&c.

Crescent Lodge, Clapham.common, April, 1841.

A woman, twenty-seven years of age, of ghort stature and spare habit, consulted me, in consequence of an occasional and slight dificulty of breathing having by degrees become frequent and distressing. The dys. pnoea was at this time very urgent, and she was unable to lie down, being compelled to maintain an upright position of the trunk both night and day. Her countenance expressed great distress, and the face was suffused and of a leaden-blue colour, and together with the neck much swollen, particu- larly on the right side. The veins of the face and nerk were greatly distended, and the upper and left side of the thorax was enlarged and tender to the touch, and the appearance of the patient was that of a person undergoing strangulation. With difficulty she stated that from childhood she had been subject to huried respiration upon even moderate exertion, and that of late years the dyspnoea had become more distressing, and she had been seized with paroxysms that threatened suffocation. She bad always felt tenderness across the upper part of the chest, and never could bear the pressure of stays or any tight article of dress. She had been married several years, had never been pregnant, and her constitution was regular. The abstraction of a few ounces of blood by venesection, at intervals of ten or twelve days, an abstemious diet, and perfect rest, alforded considerable relief for a brief period; but occasionally severe attacks of difficulty of breathing came on, and after being under my care about thee months, she suddenly expired in a paroxysm of this kind.

\section{Post.mortem Examination.}

On attempting to examine the thorax, it was impossible to reflect the sternum after dividing the ribs, in consequence of the form adhesion of a substance which was attached to the inner surface of the bones of the chest. Upon dividing, with a scalpel, the cause of the obstraction, and elevating the sternum, the thymus gland was found altered in its structure, and so much enlarged as to fill up the whole of the anterior mediastinum; it extended down to the diaphragm, and upwards as fur as the sterno-clavicular articu. lation. The greater part of the gland was of a whitish colour, and resembled in texture and appearance the udder of a cow; but some portions were of a fibro-cartilaginous structure. This morbid growth, which weighed nearly a pound, bad extended around the large vessels on the right side of the heart, and in some places was firmly adherent to the trunk of the vena cava. The obstruction to the circulation and to respiration, by the pressure of this enormous mass on the large vessels and on the trachea, must, of course, have been very great; and thas the symptoms which were present during life admitted of an easy explanation. The return of the blood from the head had long been seriously impeded, as was evinced by the blue orleaden hue of the countenance, and the turgid state of the veins; and the fatal termination of the case, was a necessary consequence of the progressive enlargement of the gland. The heart and lungs, with the exception of being much engorged with blood, exhibited no appearance of disease. The morbid parts were removed, and submitted to the examination of Sir $A$. 
Cooper and Dr. Armstrong, and were left in the possession of the latter. Both these eminent men concurred with me in the opi. nion, that the case was one of diseased and enlarged thymus gland.

\section{ON THE \\ MODE OF REDUCING LUXATIONS OF THE}

\section{INFERIOR MAXILIA.}

\section{To the Editor of THE LANCET.}

SIR :-Having been several times practically convinced of the unpleasant effects which often await the surgeon when employed in reducing dislocations of the lower jaw in the manner commonly described, I was led to inquire if the same mechanical means might not be had recourse to without the introduction of the fingers into the mouth of the patient. The chief indication in cases of this accident evidently consist in effecting its reduction, and the employment of the lower jaw as a lever of the first order; the molar teeth, upon which the thumbs of the operator are desired to be placed, acting a; the fulcrum, which is then nearly midway between the resisting and the moving power; whilst the means 1 have been induced to adopt consist in placing the fulcrum nearer to the former, and thus facilitating the mode of reduction, and also obviating the liability of the thumbs being severely bitten by the sudden and powerful elosure of the teeth, commonly consequent upon reduction. The surgeon stationing himself behind and above the patient, places the thamb of either hand upon the angle of the jaw, on a plane with the insertion of the posterior fibres of the masseter mascles, and then pressing downwards and backwards, which from his situa.. tion he can very effectually do, it only becomes necessary to elevate the symphysis of the jaw to complete the reduction.

During my house-surgeoncy at the Westminster Hospital, as well as within the last month, I have had opportunities of employing this means of reduction, and in no case has the slightest dificulty been encountered. It will be superfluous to relate all, but $I$ may be allowed to refer to three instances, one of which was extremely interesting.

Two of then occurred in the persons of women in advanced life, in whom the heads of the bones slipped from their cavities while gaping. To effect their reduction it was hardly necessary to employ any upward force on the symphysis, the condy les returning to their natural positions almost as soon as the pressure had been applied in the proper direction against the angles of the jaws.

The third was that of a young man, xt. 26, who fell through a trap-door, and struck the symphysis of the jaw with so much force as to produce its dislocation. At the time of my seeing him the masseter, tempo. ral, and pterygoid muscles had been firmly contracted, quite preventing replacement by the ordinary means; but by pressing forcibly for a short time upon the angles of the bone, and at the same time employing the rami as levers, by compressing them against the upper maxilla, the reduction was accom. plished without difficulty. Apologising for thus trespassing on your valuable time, I have the honour to be, Sir, your obedient servant,

\section{1, Broad Sanctuary, Westminster.}

Barnard Holt.

\section{ON THE \\ DIFFICULTY OF DIAGNOSIS OF HYALOIDITIS.}

\section{To the Editor of The Lancet.}

Srr:-The infrequency of death from, or daring, ophthalmic disease, and the great difficulty of obtaining a deliberate exami. nation of the orgass of vision under any circumstances, have been the obviouscauses of the incompleteness of the morbid anatony of the human eye. Hence the assump. tion of peculiar conditions of the orgun, when the subject of affections of the posterior and more hidden tissues; and hence the localising the seats of inflammation, which are neither proved by post-mortem results, nor by symptoms and appearances observed during life.

These remarks are hazarded in consequence of reading in your last Number a case of so-called hyaloiditis, by Dr. Hocker.

I by no means wish to deny that such a disease may not exist; on the contrary, any tissue organised, as the hyaloid membrane indubitably is, may be the subject of infam. matory action. I beg, therefore, simply to observe, that $I$ do not see any symptum or detailed appearance in the case of Eliz, Ward, that warrants the assumption of byaloiditis.

The case appears, from its early history, as the author justly observes, to be one of congestive amaurosis, probably congestion of both choroid and retina, subsequently proceeding to chronic inflammation of those tissues. The dark and luminous spectrd attest this fact. The eyeball enlarges, the iris is projected forwards, and the yatient becomes perfectly amaurotic. This is the result of pressure undeniably. The hard. ness of the globe, the blueness of the scle. rotic, sufficiently indicate the increased volume of fuids within the eye. Where, however, is there anything to affirm the sup. position of the pressure being on the concave surface of the retina; or, in otier words, of an increased quantity of the fluid flling the hyaloid cells. In all matters pro. 